\title{
Topographical fingerprints of many-body interference in STM junctions on thin insulating films
}

\author{
Andrea Donarini, Benjamin Siegert, Sandra Sobczyk, and Milena Grifoni \\ Institut für Theoretische Physik, Universität Regensburg, D-93040 Regensburg, Germany \\ (Received 25 June 2012; revised manuscript received 12 October 2012; published 25 October 2012)
}

\begin{abstract}
Negative differential conductance is a nonlinear transport phenomenon ubiquitous in molecular nanojunctions. Its physical origin can be the most diverse. In rotationally symmetric molecules with orbitally degenerate many-body states it can be ascribed to interference effects. We establish in this paper a criterion to identify the interference blocking scenario by correlating the spectral and the topographical information achievable in a scanning tunneling microscopy (STM) single-molecule measurement. Simulations of current-voltage characteristics as well as constant-height and constant-current STM images for a Cu-phthalocyanine on a thin insulating film are presented as experimentally relevant examples.
\end{abstract}

DOI: 10.1103/PhysRevB.86.155451

PACS number(s): 85.65.+h, 68.37.Ef, 73.63.-b

\section{INTRODUCTION}

Negative differential conductance (NDC) is a fundamental property of two-terminal devices since the discovery of the first tunnel diode. ${ }^{1}$ The realization of NDC within an atomic scale device ${ }^{2-7}$ can consequently be regarded as a milestone in the process of miniaturization which drives the information technology.

Scanning tunneling microscopy (STM) experiments have played an important role in this research field, giving several examples of NDC observed with a variety of nanojunctions. A number of physical scenarios have been proposed for the explanation of the experimental findings: among others, the existence of sharp resonances on both electrodes, ${ }^{3,8}$ the voltage-dependent increase in the tunneling barrier height, ${ }^{9,10}$ the orbital matching between molecule and tip, ${ }^{11,12}$ or even just the symmetry matching between surface states in the substrate and molecular states. ${ }^{13}$ Last but not least, vibrational-mediated NDC has also been observed in single-molecule devices ${ }^{14}$ and proposed to test position-dependent Franck-Condon factors in suspended carbon nanotubes. ${ }^{15}$

Recently also interference phenomena in single-molecule junctions have attracted intense theoretical ${ }^{16-27}$ and experimental $^{28-31}$ investigations. These junctions allow us to tackle the fundamental question of the quantum mechanical nature of the electronic transport at the nanoscale and exhibit dramatic modulations of the current desirable for applications. The quest for specific fingerprints of the electronic interference which go beyond the bare current or conductance suppression $^{30}$ remains, though, a crucial issue. We establish in this article a criterion to identify the interference blocking scenario by correlating the spectral and the topographical information achievable in an STM single-molecule measurement (see Figs. 1-3).

In a recent publication we predicted the occurrence of NDC due to interference blocking ${ }^{21-23}$ in an STM single benzene junction on a thin insulating film. ${ }^{32}$ Benzene, however, is not easily accessible in STM experiments and it is not obvious to what extent the findings of Ref. 32 apply to larger, experimentally relevant molecules (see, e.g., Refs. 33 and 34). A major result of this article is an analytical expression for the current as a function of the applied bias voltage encompassing various transport regimes [see Eqs. (11) and (12)], which provides both the criteria for the occurrence of interference blocking NDC and the interpretation of its topographical fingerprints. Specifically, interference NDC is expected to occur in molecules which exhibit an electron affinity (ionization potential) $E_{0}-E_{1}\left(E_{-1}-E_{0}\right)$ very close to the work function $\phi_{0}$ of the substrate and, due to their rotational symmetry, have an orbitally degenerate anion (cation) many-body eigenstate (here $E_{0}$ and $E_{ \pm 1}$ denote the many-body ground-state energy of the neutral molecule and of the anion or cation). The necessary decoupling from the substrate, originally obtained through a thin insulating layer, ${ }^{33}$ can also be achieved by combining two different molecules in a monolayer directly adsorbed on a metal surface. ${ }^{7}$ Recently, this setup has been used to demonstrate position-dependent local gating, ${ }^{35}$ thus suggesting an alternative possibility to achieve the mentioned interference conditions. Topographical fingerprints to identify the interference blocking scenario are predicted for both the constant-height and the constant-current scanning modes. Interference is associated, in the first case, with a flattening of the current map in the molecule region, with a corresponding loss of the characteristic nodal plane pattern (see Fig. 3), and, in the second case, with an enhanced sensitivity of the apparent height of the molecule to the operating current (see Fig. 6). As we will prove later, both phenomena have the same origin; i.e., in the interference blocking regime, the bottleneck process defining the current pattern is a substrate and not a tip tunneling event. The analytical results apply to a wide class of molecular junctions. In particular, we present simulations concerning a CuPc junction.

\section{MODEL}

We describe the STM single-molecule junction as a systembath model,

$$
H=H_{\text {mol }}+H_{\text {sub }}+H_{\text {tip }}+H_{\text {tun }},
$$

where $H_{\mathrm{mol}}$ is the Hamiltonian for the isolated molecule, in which, to fix the ideas, we distinguish a single-particle component $H_{0}$ and a two-particle component $V$, both expressed in terms of creation and annihilation operators $\left\{d_{\alpha \sigma}, d_{\alpha \sigma}^{\dagger}\right\}$ for the atomic orbitals $\psi_{\alpha}$, where $\alpha$ indicates both the site and the atomic species. $H_{\text {sub }}$ and $H_{\text {tip }}$ account for the substrate and the tip, respectively, which we assume as reservoirs of noninteracting electrons with different spatial confinement. Finally, $H_{\text {tun }}$ describes the tunneling coupling between the 


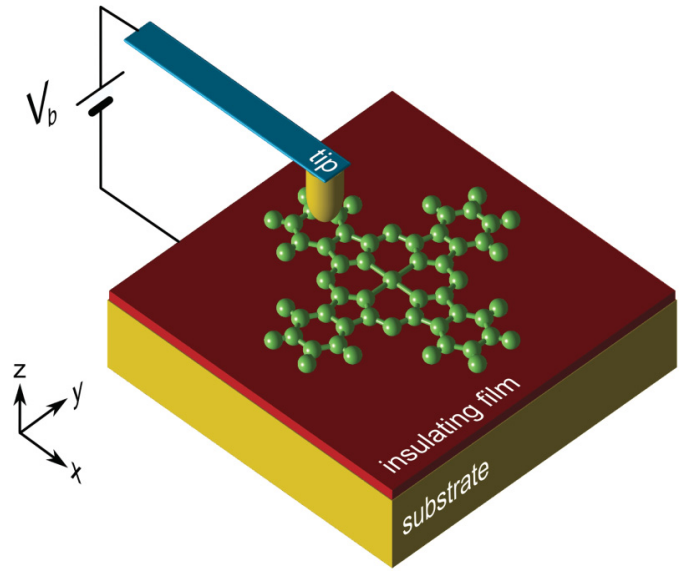

FIG. 1. (Color online) Artistic view of an STM single-molecule junction. We show in yellow (light gray) the metallic leads (tip and substrate), in red (dark gray) the thin insulating film, and in green (medium gray) the schematic representation of a $\mathrm{CuPc}$.

metallic leads and the molecule:

$$
H_{\mathrm{tun}}=\sum_{\chi k \ell m \sigma} t_{k \ell m}^{\chi} c_{\chi k \sigma}^{\dagger} d_{\ell m \sigma}+\text { H.c., }
$$

where $\chi=S, T$ indicates the substrate or the tip, $k$ the momentum, and $\sigma$ the spin of the electron in the lead. Due to their rotational symmetry, the molecular orbitals are classified using the projection $\ell$ of the angular momentum along the principal rotation axis of the molecule. A further quantum number $m$ is introduced to account for possible degeneracies in the spectrum of the angular momentum. Finally, the tunneling amplitudes $t_{k \ell m}^{\chi}$ take the form

$$
\begin{aligned}
t_{\vec{k} \ell m}^{\mathrm{S}} & =\varepsilon_{\ell m}\langle S \vec{k} \sigma \mid \ell m \sigma\rangle, \\
t_{k_{z} \ell m}^{\mathrm{T}} & =\varepsilon_{\ell m}\left\langle T k_{z} \sigma \mid \ell m \sigma\right\rangle,
\end{aligned}
$$

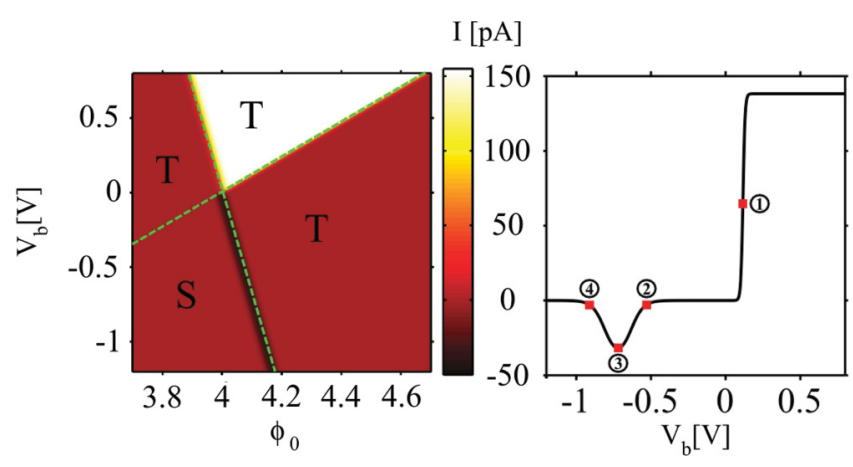

FIG. 2. (Color online) Left: Current through a CuPc singlemolecule junction as a function of the substrate (and tip) work function $\phi_{0}$ and of the sample bias $V_{b}$. The tip apex position is set at $(x, y, z-d)=(+5,-5,7) \AA$, with the origin taken on the metal-insulator interface and in correspondence with the center of the molecule and with $d$ being the thickness of the insulating layer (see Fig. 1). The tip and substrate resonant lines (with positive and negative slopes, respectively) divide the parameter space into four regions. T (S) indicates a region in which the current is proportional to the tip (substrate) tunneling rate. Right: Current obtained from a cut of the left-hand plot corresponding to $\phi_{0}=4.1 \mathrm{eV}$. The numbers in the current-voltage plot refer to the current maps in Fig. 3. The current scale is the same for the left and right panels.

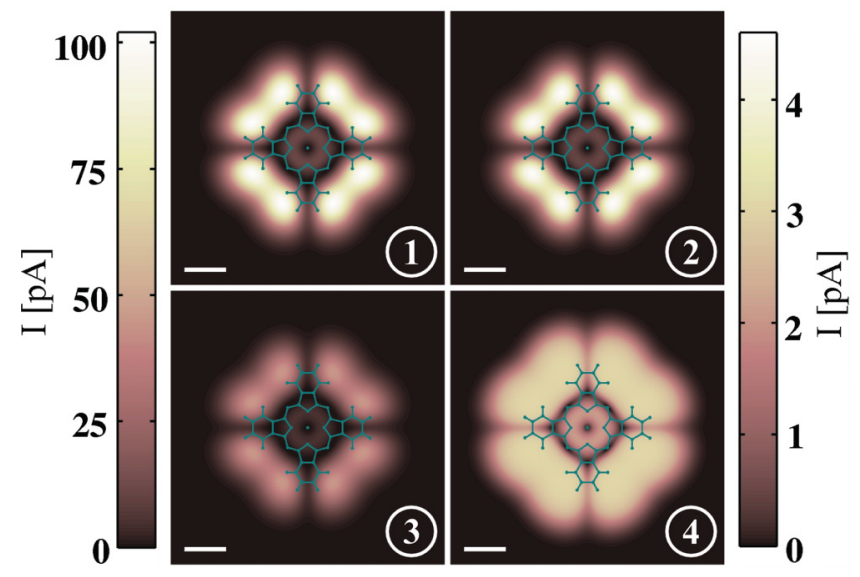

FIG. 3. (Color online) Constant-height current maps calculated for different bias voltages. The color bar on the left (right)-hand side corresponds to maps 1 and 3 (maps 2 and 4). The 5-§̊-long white line sets the scale of the images. The numbers in the maps refer to the biases indicated in the right panel in Fig. 2. The current map in the interference blockade regime (map 4) appears to be flat in the molecule region. The characteristic nodal plane pattern appears instead to be much more pronounced at the positive and negative bias resonances (maps 1 and 3) and even in the Coulomb blockade region (map 2). The tip apex is placed at $7 \AA$ above the molecular plane, while the substrate biases are, respectively $V_{b 1}=0.1153 \mathrm{~V}$, $V_{b 2}=-0.5303 \mathrm{~V}, V_{b 3}=-0.7201 \mathrm{~V}$, and $V_{b 4}=-0.9118 \mathrm{~V}$.

where $\varepsilon_{\ell m}$ is the energy eigenvalue of the single-particle Hamiltonian $H_{0}$ associated with state $|\ell m \sigma\rangle$. For the substrate and tip states we assume the model described in Ref. 32: a three-dimensional momentum is necessary for the extended substrate states, while only the momentum in the transport direction characterizes the tip states which are confined in the $x$ and $y$ direction around the tip position.

Our method of choice for treating the dynamics in the regime of weak coupling between system and leads is the Liouville equation method. We start from the Liouville equation for the total density operator $\rho(t)$ of the whole system, consisting of the molecule, the tip, and the substrate. We focus on the time evolution of the reduced density operator $\sigma=\operatorname{Tr}_{\mathrm{S}+T}\{\rho\}$, formally obtained by taking the trace over the unobserved degrees of freedom of the tip and the substrate. A detailed discussion and derivation of the equation of motion for the reduced density operator of the system can be found, e.g., in Refs. 36 and 21, and its adaptation to the STM setup on thin insulating films in Ref. 32. For a general discussion about the reduced density matrix and related equations of motion, see also Refs. 37 and 38.

Let us now consider a molecule deposited on a substrate having a nondegenerate neutral ground state $\mid \mathrm{N} E_{0} \ell=\ell_{0} S_{z}=$ $0\rangle$ and an orbitally degenerate anion ground state $\mid \mathrm{N}+1 E_{1} \ell=$ $\left.\pm \ell_{1} S_{z}= \pm 1 / 2\right\rangle$. Assume that the neutral state minimizes the grand canonical Hamiltonian $H_{\mathrm{G}}=H-\mu_{0} N$, where $\mu_{0}=-\phi_{0}$ is the equilibrium chemical potential of the leads, $\phi_{0}$ the corresponding work function, and $\mathrm{N}$ the electron number operator for the molecule. If $E_{1}-E_{0} \approx \mu_{0}$, there is a bias window in which the transport characteristics are dominated by a dynamics which involves the neutral and 
(a)

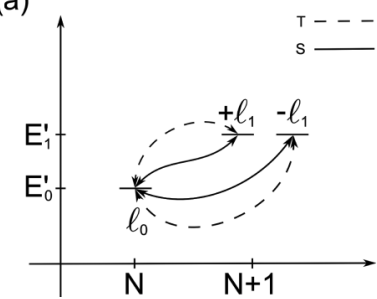

(b)

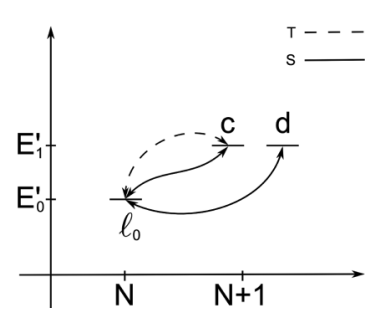

FIG. 4. Schematic representation of the many-body states participating in the transport. On the vertical axis we report the grand canonical energies $E_{0}^{\prime}:=E_{0}-\mathrm{N} \mu_{0}$ and $E_{1}^{\prime}:=E_{1}-(\mathrm{N}+$ 1) $\mu_{0}$, where $\mu_{0}$ is the equilibrium chemical potential for the leads. (a) We adopt the angular momentum representation; (b) the decoupling basis is introduced for the anionic states (see text for details).

anionic ground states only. In Fig. 4(a) we give a schematic representation of the many-body states participating in the transport and the associated transition rates, where, for the sake of simplicity, we neglect the spin degree of freedom. According to the general theory presented in [32], the corresponding generalized master equation for the reduced density matrix in the angular momentum basis reads

$$
\begin{aligned}
\dot{\sigma}_{\ell_{0} \ell_{0}}^{\mathrm{N} E_{0}}= & -\sum_{\chi \tau \ell} R_{\ell-\ell_{0}, \ell-\ell_{0}}^{\chi \tau}(\Delta E) f_{\chi}^{+}(\Delta E) \sigma_{\ell_{0} \ell_{0}}^{\mathrm{N} E_{0}} \\
& +\sum_{\chi \tau \ell \ell^{\prime}} R_{\ell-\ell_{0}, \ell^{\prime}-\ell_{0}}^{\chi \tau}(\Delta E) f_{\chi}^{-}(\Delta E) \sigma_{\ell^{\prime} \ell}^{\mathrm{N}+1 E_{1} \tau} \\
\dot{\sigma}_{\ell \ell^{\prime}}^{\mathrm{N}+1 E_{1} \tau}= & -\frac{1}{2} \sum_{\chi \ell^{\prime \prime}}\left[R_{\ell-\ell_{0}, \ell^{\prime \prime}-\ell_{0}}^{\chi \tau}(\Delta E) \sigma_{\ell^{\prime \prime} \ell^{\prime}}^{\mathrm{N}+1 E_{1} \tau},\right. \\
& \left.\sigma_{\ell \ell^{\prime \prime}}^{\mathrm{N}+1 E_{1} \tau} R_{\ell^{\prime \prime}-\ell_{0}, \ell^{\prime}-\ell_{0}}^{\chi \tau}(\Delta E)\right] f_{\chi}^{-}(\Delta E) \\
& +\sum_{\chi \tau} R_{\ell-\ell_{0}, \ell^{\prime}-\ell_{0}}^{\chi \tau}(\Delta E) f_{\chi}^{+}(\Delta E) \sigma_{\ell_{0} \ell_{0}}^{\mathrm{N} E_{0}},
\end{aligned}
$$

where $\ell, \ell^{\prime}$, and $\ell^{\prime \prime}= \pm \ell_{1}$, span the angular momenta of the anionic ground state and $\Delta E=E_{1}-E_{0}$ is the energy difference between the anionic and the neutral ground states. Moreover, $f_{\chi}^{+}(x)$ is the Fermi function for the lead $\chi$, $f_{\chi}^{+}(x):=f\left(x-\mu_{\chi}\right)$, and $f_{\chi}^{-}(x):=1-f_{\chi}^{+}(x)$. Note that we assume an asymmetric potential drop where $\mu_{T}=\mu_{0}-c e \mathrm{~V}_{b}$ with $c=0.86$ and $\mu_{S}-\mu_{T}=e \mathrm{~V}_{b}$. The rate $R_{\ell-\ell_{0}, \ell^{\prime}-\ell_{0}}^{\chi \tau}$ is

defined as

$$
\begin{aligned}
R_{\Delta \ell, \Delta \ell^{\prime}}^{\chi \tau}(\Delta E)= & \sum_{m m^{\prime}}\left\langle\mathrm{N}+1 E_{1} \ell \tau\left|d_{\Delta \ell m \tau}^{\dagger}\right| \mathrm{N} E_{0} \ell_{0} 0\right\rangle \\
& \times \Gamma_{\Delta \ell m, \Delta \ell^{\prime} m^{\prime}}^{\chi}(\Delta E) \\
& \times\left\langle\mathrm{N} E_{0} \ell_{0} 0\left|d_{\Delta \ell^{\prime} m^{\prime} \tau}\right| \mathrm{N}+1 E_{1} \ell^{\prime} \tau\right\rangle,
\end{aligned}
$$

where

$$
\Gamma_{\Delta \ell m, \Delta \ell^{\prime} m^{\prime}}^{\chi}(\Delta E)=\frac{2 \pi}{\hbar} \sum_{k}\left(t_{k \Delta \ell m}^{\chi}\right)^{*} t_{k \Delta \ell^{\prime} m^{\prime}}^{\chi} \delta\left(\varepsilon_{k}^{\chi}-\Delta E\right),
$$

and we have introduced the notation $\Delta \ell=\ell-\ell_{0}, \Delta \ell^{\prime}=\ell^{\prime \prime}-$ $\ell_{0}$ for the variation in angular momenta associated with the tunneling process.

Due to the rotational symmetry of the molecule and the different spatial confinement of the leads, the rate matrices acquire the form

$$
\begin{aligned}
& R_{\Delta \ell, \Delta \ell^{\prime}}^{S}=R^{S} \delta_{\Delta \ell, \Delta \ell^{\prime}}, \\
& R_{\Delta \ell, \Delta \ell^{\prime}}^{T}=R^{T} \exp \left(-\mathrm{i} \frac{\Delta \ell-\Delta \ell^{\prime}}{\Delta \ell} \phi_{\Delta \ell}\right),
\end{aligned}
$$

where, for simplicity, we did not write the energy dependence of $R^{S}$ or the energy and tip position dependence of $R^{T}$ and of the phase $\phi_{\Delta \ell}$. Moreover, the latter is defined as

$$
\phi_{\Delta \ell}=\arg \left(\sum_{m} t_{\tilde{k} \Delta \ell m}^{T}\left\langle\mathrm{~N} E_{0} \ell_{0} 0\left|d_{\Delta \ell m \tau}\right| \mathrm{N}+1 E_{1} \ell \tau\right\rangle\right) .
$$

Due to their particular structure, the rate matrices, (7), are both diagonalized by the same basis transformation.

While the substrate rate matrix is invariant under whatever unitary transformation, the tip rate matrix acquires a peculiar diagonal form since one of its eigenvalues vanishes. The basis transformation, within each spin sector of the anionic ground state, reads

$$
\left(\begin{array}{l}
|\mathrm{c}\rangle \\
|\mathrm{d}\rangle
\end{array}\right)=\frac{1}{\sqrt{2}}\left(\begin{array}{cc}
e^{-\mathrm{i} \phi_{\Delta \ell}} & e^{+\mathrm{i} \phi_{\Delta \ell}} \\
e^{-\mathrm{i} \phi_{\Delta \ell}} & -e^{+\mathrm{i} \phi_{\Delta \ell}}
\end{array}\right)\left(\begin{array}{c}
\left|+\ell_{1}\right\rangle \\
\left|-\ell_{1}\right\rangle
\end{array}\right)
$$

and it depends on the position of the tip via the phase $\phi_{\Delta \ell}$. Due to the diagonal form of the rate matrices, in this basis the dynamics is described only by means of populations. In particular, the decoupled states $\left|\mathrm{N}+1 E_{1} \mathrm{~d} \tau\right\rangle$ are only coupled to the neutral ground state $\left|N E_{0} \ell_{0} 0\right\rangle$ via substrate-molecule tunneling events. Both tunneling couplings are still open instead for the coupled states $\left|\mathrm{N}+1 E_{1} \mathrm{c} \tau\right\rangle$ [see Fig. 4(b)]. The corresponding master equation reads

$$
\left(\begin{array}{c}
\dot{\sigma}^{\mathrm{N}} \\
\dot{\sigma}_{\mathrm{c}}^{\mathrm{N}+1 \tau} \\
\dot{\sigma}_{\mathrm{d}}^{\mathrm{N}+1 \tau}
\end{array}\right)=\left[2 R^{T}\left(\begin{array}{ccc}
-2 f_{T}^{+} & 2 f_{T}^{-} & 0 \\
f_{T}^{+} & -f_{T}^{-} & 0 \\
0 & 0 & 0
\end{array}\right)+R^{S}\left(\begin{array}{ccc}
-4 f_{S}^{+} & 2 f_{S}^{-} & 2 f_{S}^{-} \\
f_{S}^{+} & -f_{S}^{-} & 0 \\
f_{S}^{+} & 0 & -f_{S}^{-}
\end{array}\right)\right]\left(\begin{array}{c}
\sigma^{\mathrm{N}} \\
\sigma_{\mathrm{c}}^{\mathrm{N}+1 \tau} \\
\sigma_{\mathrm{d}}^{\mathrm{N}+1 \tau}
\end{array}\right),
$$

where, for simplicity, we have omitted the arguments $(\Delta E)$ of the Fermi functions and the tunneling rates $R^{\chi}$ and suppressed the indexes $E_{0}, \ell_{0}$, and $E_{1}$ in the elements of the density matrix.
The stationary current flowing through the STM junction is calculated as the average $\left\langle I_{S}\right\rangle=\operatorname{Tr}\left\{\sigma^{\text {stat }} I_{S}\right\}=-\left\langle I_{T}\right\rangle$, where $\sigma^{\text {stat }}$ is the stationary solution of Eq. (10) and $I_{\chi}$ are the current 
operators, which are directly obtained from Eq. (4) following, for example, Refs. 21 and 32. Despite its simplicity, Eq. (10) describes the system in a variety of different regimes which leave their fingerprints in the current-voltage characteristics and in the corresponding STM images.

\section{RESULTS}

Given Eq. (10), the stationary current flowing through the system is found in closed analytical form, which represents one major result of this work. It reads

$$
I\left(\vec{R}_{\text {tip }}, V_{\mathrm{b}}\right)=2 e R^{S} f_{S}^{+} \sigma^{\mathrm{N}}\left(1-\frac{\sigma_{\mathrm{c}}^{\mathrm{N}+1 \tau}}{\sigma_{\mathrm{d}}^{\mathrm{N}+1 \tau}}\right),
$$

where $e$ is the (negative) electron charge, and for the stationary populations, it holds that

$$
\begin{aligned}
\sigma^{\mathrm{N}} & =\left(1+2 \frac{R^{S} f_{S}^{+}+2 R^{T} f_{T}^{+}}{R^{S} f_{S}^{-}+2 R^{T} f_{T}^{-}}+2 \frac{f_{S}^{+}}{f_{S}^{-}}\right)^{-1}, \\
\frac{\sigma_{\mathrm{c}}^{\mathrm{N}+1 \tau}}{\sigma_{\mathrm{d}}^{\mathrm{N}+1 \tau}} & =\frac{R^{S} f_{S}^{+}+2 R^{T} f_{T}^{+}}{R^{S} f_{S}^{-}+2 R^{T} f_{T}^{-}} \cdot \frac{f_{S}^{-}}{f_{S}^{+}} .
\end{aligned}
$$

Depending on the rate $R^{T}$ at the tip position and the bias and the Fermi functions at the bias, both topographical and spectral information is embedded in Eq. (11).

In the right panel in Fig. 2 we report the $I V$ characteristics calculated for a $\mathrm{Cu}$-phthalocyanine on a metal-insulator substrate (a 7- $\AA$-thick insulator with relative dielectric constant $\left.\varepsilon_{\mathrm{r}}=5.9\right)$ with an effective work function $\phi_{S}=4.1 \mathrm{eV}$. We set up the single-particle Hamiltonian for the molecule in the tight-binding approximation and calculate the hopping terms following the Slater-Koster scheme. ${ }^{39}$ Moreover, we adopt the constant interaction approximation and assume a charging energy that fits the experimentally evaluated electron affinity $E_{0}-E_{1}$ of CuPc of $4 \mathrm{eV}$.

At a low bias the current is suppressed by Coulomb blockade. As the bias increases on the positive side (conventionally under this condition electrons flow from the tip to the substrate), the current undergoes a sudden jump corresponding to the opening of the neutral-anion transition at the tip-molecule interface $\left(E_{1}-E_{0}=\mu_{T}\right)$. On the negative bias side the Coulomb blockade is also lifted, but this time at the substrate resonance point $\left(E_{1}-E_{0}=\mu_{S}\right)$, and the current shows a sharp peak whose width scales with the temperature $\left(k_{\mathrm{B}} T=6 \mathrm{meV}\right.$ in all presented plots). At higher negative biases the current is blocked due to interference and the decoupled anionic state is the sink of the system. A crucial condition for the interference blocking to occur is that $E_{1}-E_{0} \ll E_{0}-E_{-1}$, ensuring that the substrate-molecule anion resonance anticipates the tip-molecule cation one, which would otherwise dominate the transport characteristics.

Analogous interference blocking involving degenerate many-body states has been encountered in a variety of systems. ${ }^{19,21-23}$ Nevertheless, the STM setup described here uniquely allows us to correlate the interference current blocking with specific topographical fingerprints. In Fig. 3 we present different constant-height current maps (the tip is always positioned $7 \AA$ above the molecular plane) corresponding to the different points labeled in the right panel in Fig. 2. Maps 1

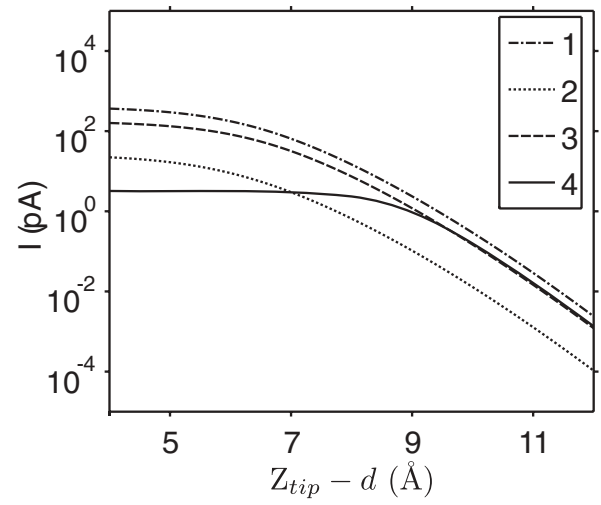

FIG. 5. Current vs tip-molecule distance calculated for different biases. Numbers in the legend correspond to the different cases illustrated in Fig. 3: $V_{b 1}=0.1153 \mathrm{~V}, V_{b 2}=-0.5303 \mathrm{~V}, V_{b 3}=$ $-0.7201 \mathrm{~V}$, and $V_{b 4}=-0.9118 \mathrm{~V}$. Note, in particular, the wide plateau associated with the interference blockade regime (line 4) and its crossing with the Coulomb blockade line for $Z_{\text {tip }}-d=7 \AA$.

and 3 are calculated for the tip and substrate resonant tunneling conditions, and maps 2 and 4 for the Coulomb and interference blockade regimes, respectively. Striking is the flattening of the current map obtained in the interference case (map 4) when compared to all other regimes.

Signatures of interference can be clearly seen also in the current vs tip-molecule distance represented in Fig. 5. The four traces correspond to the four different bias conditions indicated by the numbers 1 to 4 in the right panel in Fig. 2 and the tip is in the same $x y$ position. At large tip-molecule distances all traces show the exponentially decaying behavior typical of the STM measurements (roughly 1 order of magnitude decay per $\AA$ ). At shorter distances, all curves saturate due to the form of the $p_{z}$ orbitals. Contrary to the others, though, the curve corresponding to the interference blockade regime (case 4) saturates at larger distances and shows a wide plateau. For this reason it even crosses the Coulomb blockade trace (case 2) at a tip-molecule distance of $7 \AA$, consistent with the result in Fig. 2.

Finally, we also present in Fig. 6 several constant-current topographic maps simulated for different biases and different operating currents. The surfaces presented in the upper panel correspond to the Coulomb blockade regime; the ones in the lower panel, to the interference blockade. Due to the particular choice of the biases, the apparent height of the molecule is exactly the same if we choose $3 \mathrm{pA}$ as an operating current. The shape of the molecule is not modified in the interference blockade regime, as it is for the constant-height current maps (see Fig. 3). Yet, the enhanced sensitivity of the apparent height of the molecule with respect to the variation of the operating current, when compared with the same measurement in the Coulomb blockade regime. The surfaces presented in Fig. 6 correspond, in fact, for both cases, to operating currents in the range $2.85-3.15 \mathrm{pA}$.

\section{DISCUSSION}

All the results presented in the previous section can be understood by analyzing the different limits of Eq. (11). Let us first consider the Coulomb blockade regime. The latter is 

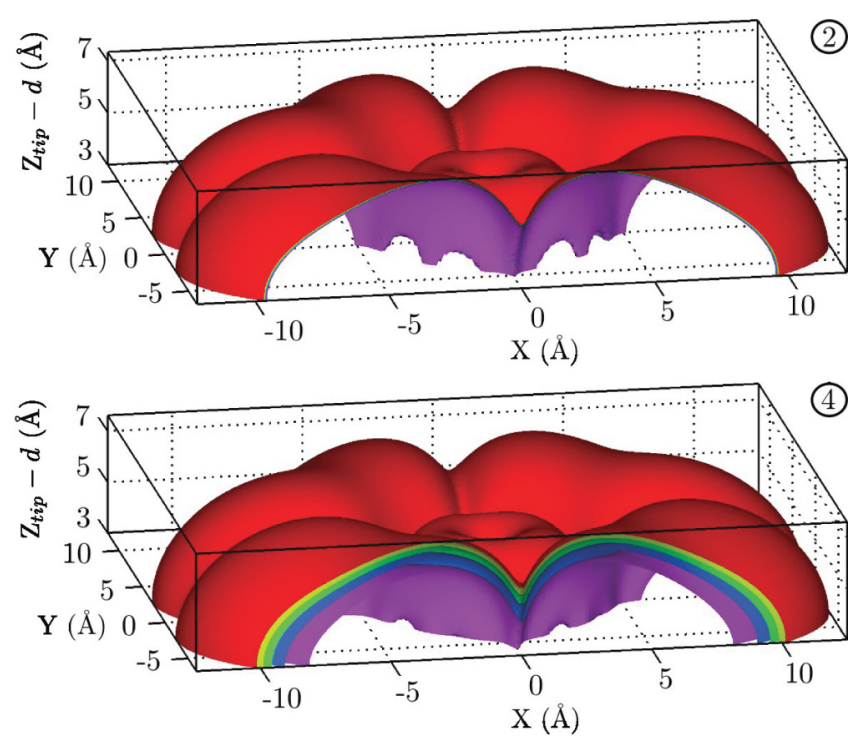

FIG. 6. (Color online) Isosurfaces of constant current calculated in the proximity of the Coulomb blockade (upper panel; $V_{b}=$ $-0.5303 \mathrm{~V}$ ) and interference blockade (lower panel; $V_{b}=-0.9118$ V) regimes. The surfaces correspond, in both cases, to the currents: $I=3.15,3.075,3.0,2.925$, and $2.85 \mathrm{pA}$.

defined, for $V_{\mathrm{b}}<0$, by the inequality $E_{1}-E_{0}-\mu_{\mathrm{S}} \gg k_{\mathrm{B}} T$, which in turn implies $f_{\mathrm{S}}^{+} \ll 1$ and $f_{\mathrm{T}}^{+} \ll 1$. In this limit and under the asymmetry relation $R^{T} \ll R^{S}$ typical of an STM experiment, it is not difficult to prove that

$$
I_{\mathrm{CB}}=4 e R^{T} f_{T}^{-} \frac{f_{S}^{+}}{f_{S}^{-}}\left(1+4 \frac{f_{S}^{+}}{f_{S}^{-}}\right)^{-1} \approx 4 e R^{T} f_{S}^{+} .
$$

The current is thus proportional to the tip rate. The equality in Eq. (13) also has a precise physical interpretation. The charge fluctuations at the substrate lead represent the fastest phenomenon $\left(f_{S}^{+} / f_{T}^{+} \gg 1\right.$ due to the asymmetric potential drop at tip-molecule and substrate-molecule contacts) which sets the ratio between the populations of the states to be the thermal average, $\sigma_{\mathrm{c} / \mathrm{d}}^{\mathrm{N}+1 \tau} / \sigma^{N}=f_{S}^{+} / f_{S}^{-}$. Finally, the trace sum rule implies

$$
\sigma^{\mathrm{N}}=\left(1+4 \frac{f_{S}^{+}}{f_{S}^{-}}\right)^{-1}
$$

The current is determined instead by the slowest process: the tunneling event $\left|\mathrm{N}+1 E_{1} \mathrm{c} \tau\right\rangle \rightarrow\left|\mathrm{N} E_{0} 0\right\rangle$ towards the tip. Equation (13) follows due to the presence of two spin channels and the fact that the tip rate for the coupled state is $2 R^{T}$. Analogously, for $V_{\mathrm{b}}>0$, the Coulomb blockade condition reads $E_{1}-E_{0}-\mu_{T} \gg k_{\mathrm{B}} T$ and the current is again proportional to the tip rate, namely, $I=-4 e R^{T} f_{T}^{+}$. Thus, the constant-height current map reproduces the shape of the molecular orbital encoded in $R^{T}$.

The interference blockade regime is confined to the negative bias and it is defined by the inequality $E_{1}-E_{0}-\mu_{S} \ll$ $-k_{\mathrm{B}} T$, which implies $f_{S}^{+} \approx 1$ and $f_{T}^{+} \ll 1$. Under these conditions the current, Eq. (11), reduces to

$$
I_{\mathrm{IB}}=e \frac{R^{S} f_{S}^{-} R^{T} f_{T}^{-}}{R^{S} f_{S}^{-}+R^{T} f_{T}^{-}} .
$$

Equation (15) tells us even more clearly, when cast in the form $I_{\mathrm{IB}}^{-1}=\left(e R^{S} f_{S}^{-}\right)^{-1}+\left(e R^{T} f_{T}^{-}\right)^{-1}$, that the current is the result of two competing processes happening in series: the thermal unblocking of the decoupled state $\left|\mathrm{N}+1 E_{1} \mathrm{~d} \tau\right\rangle \rightarrow$ $\left|\mathrm{N} E_{0} 0\right\rangle$ towards the substrate and the tip tunneling event $\left|\mathrm{N}+1 E_{1} \mathrm{c} \tau\right\rangle \rightarrow\left|\mathrm{N} E_{0} 0\right\rangle$. Note that in the system dynamics the two tunneling events are not independent: one cannot happen if the other did not happen before. In the interference blocking regime $f_{S}^{-} \ll f_{T}^{-}$, but in an STM set up, it typically also holds that $R^{T} \ll R^{S}$. To fix the ideas let us first fix the tip position (thus, the ratio $R^{T} / R^{S}$ ) and lower the bias, deep in the interference blockade, so as to fulfill the condition $R^{S} f_{S}^{-} \ll R^{T} f_{T}^{-}$. The current is thus proportional to $R^{S}$ and independent of the tip position. This fact explains the flattening of the constant-height current map in Fig. 3 and the wide plateau of the current vs tip-molecule distance in Fig. 5. Nevertheless, as the tip moves far from the molecule, the tip rate drops and, as the condition $R^{T} f_{T}^{-} \ll R^{S} f_{S}^{-}$is fulfilled, the position dependence of the current is recovered $\left(I_{\mathrm{IB}} \propto R^{T}\right)$. The crossover between the two regimes is estimated by the relation

$$
R^{T}\left(\vec{R}_{\mathrm{tip}}, \Delta E\right)=R^{S} e^{\beta\left(\Delta E-\mu_{S}\right)} .
$$

with $\beta=\left(k_{\mathrm{B}} T\right)^{-1}$. For completeness we add that the interference blockade is the only regime in which the current loses its canonical dependence on the tip position: the current in fact saturates to $I=-4 e R^{T}$ for large positive biases; it is $I=-2 e R^{T}$ at the tip-molecule resonance and $I=\frac{4}{5} e R^{T}$ at the substrate-molecule resonance. The summary of these results and their extension to the work function and bias voltage plane is presented in the left panel in Fig. 2, where the letters $\mathrm{T}$ and $\mathrm{S}$ indicate regions where the current is proportional to the tip or substrate rate, respectively.

The enhanced sensitivity of the apparent molecular height to the value of the operating current of a constant-current scan performed in the interference blocking regime can also be explained by analyzing Eqs. (13) and (15). Let us consider a certain value for the operating current $I_{0}$. The equations for the constant-current isosurfaces read

$$
\begin{aligned}
& R^{T}=\frac{I_{0}}{4 e f_{S}^{+}} \equiv K_{\mathrm{CB}}\left(I_{0}, V_{b}\right), \\
& R^{T}=\frac{I_{0}}{e f_{T}^{-}}\left(1-\frac{I_{0}}{e R^{S} f_{S}^{-}}\right)^{-1} \equiv K_{\mathrm{IB}}\left(I_{0}, V_{b}\right),
\end{aligned}
$$

respectively, for the Coulomb blockade and interference blockade regimes. If, for a given choice of the parameters $I_{0}$ and $V_{b}$, it holds that $K_{\mathrm{IB}}=K_{\mathrm{CB}}$, the two associated constantcurrent isosurfaces coincide. This is indeed, by construction, the case for the bias corresponding to points 2 and 4 in Fig. 2 if the operating current is chosen exactly as the one in the $I V$ characteristics (3 pA). Nevertheless, for the same choice of biases, a very different sensitivity of the constant-current isosurface to the value of the operating current is shown in the interference blockade and in the Coulomb blockade cases (compare upper vs lower panel in Fig. 6). By analyzing the second equation in (17) we can see that $K_{\mathrm{IB}}$ diverges for $I_{0}$ in the vicinity of the interference current $e R^{S} f_{S}^{-}$, while $K_{\mathrm{CB}}$ shows, in the same limit, a completely regular behavior. As $K_{\mathrm{IB}} \rightarrow \infty$ the corresponding isosurface shrinks 
rapidly as shown in Fig. 6. Moreover, the interference current also represents, in the vicinity of the interference blockade regime, an upper limit for the operating current accessible to a constant-current STM scan. In fact, for $I_{0}>e R^{S} f_{S}^{-}$the constant $K_{\mathrm{IB}}$ turns negative and the second equation in (17) cannot be fulfilled for any position of the tip.

Special consideration should be given to the robustness of the presented effect. Indeed we have so far presented the idealized situation in which the rotational symmetry of the $\mathrm{CuPc}$ is assumed to be unperturbed, with a consequently perfect degeneracy of the anion ground states. Nevertheless, this perfect degeneracy is not a necessary condition for the occurrence of the many-body interference effect described in this article. As we explicitly showed in a previous publication, ${ }^{21}$ the interference blocking scenario persists as long as the quasidegeneracy is present, i.e., the splitting of the interfering energy levels is smaller than the tunneling coupling. In fact, if the tunneling coupling is strong enough, the indetermination principle does not allow us to distinguish between the two quasidegenerate states in the tunneling event and interference takes place. Moreover, since the tip tunneling coupling is controlled, in an STM experiment, by the tip position, the interference between quasidegenerate states could also be controlled by the tip position. The result would be the tuning, with the tip-molecule distance, of the NDC at negative bias voltages associated with the interference blocking. Finally, concerning the effect of the substrate on the molecular symmetry, we would like to mention that a strong experimental sensitivity to molecular symmetry has been proven for derivatives of $\mathrm{CuPc}$ molecules on thin insulating films (see Ref. 40), suggesting that an almost-complete decoupling of the molecular states is indeed a good approximation for these systems.

Finally, the results presented so far for $\mathrm{CuPc}$ apply in general to the class of planar molecules belonging to the $C_{\mathrm{nv}}$ symmetry group, i.e., invariant under the set of rotations of angles $z 2 \pi / n, z=0, \ldots, n-1$ around a principal rotation axis perpendicular to the molecular plane and to a set of $n$ vertical planes (see Fig. 1). Their many-body states, like the single-particle ones, can be classified using the projection $\ell$ of the angular momentum in the direction of the main rotational axis (conventionally the $z$ axis) that we introduced in Eq. (2). The generic many-body eigenstates of $H_{\mathrm{m}}$ can thus be written in the form $\left|\mathrm{N} E \ell S_{z}\right\rangle$, where $\mathrm{N}$ is the particle number, $E$ the energy, and $S_{z}$ and $\ell$ the projections of the total spin and of the angular momentum in the $z$ direction, respectively, in units of $\hbar$. The state $\left|\mathrm{N} E \ell S_{z}\right\rangle$ transforms under a rotation of an angle $\phi=z 2 \pi / n$ around the main rotation axis as

$$
R_{\phi}\left|\mathrm{N} E \ell S_{z}\right\rangle=e^{\mathrm{i} \phi\left(\ell+S_{z}\right)}\left|\mathrm{N} E \ell S_{z}\right\rangle
$$

where $R_{\phi}$ is the rotation operator. Consequently, it is not difficult to prove that $\ell$ is an integer number and $-\frac{n}{2}<\ell \leqslant \frac{n}{2}$ for $C_{\mathrm{nv}}$ molecules with even $n$ and $-\frac{n-1}{2} \leqslant \ell \leqslant \frac{n-1}{2}$ when $n$ is odd. Since $C_{\mathrm{nv}}$ admits, at a maximum, bidimensional irreducible representations, we conclude that states with opposite $\ell$, connected by the reflection operation through the $n$ vertical planes, have symmetry-protected degeneracy and only states with $\ell=0$ (for even or odd $n$ ) or $\ell=0, \frac{n}{2}$ (for even $n$ ) are nondegenerate.

\section{CONCLUSIONS}

By studying the transport characteristics of an STM singlemolecule junction on a thin insulating film, we identify in this article a class of molecules that should present strong NDC and interference blocking features. Moreover, we establish a criterion to identify the interference blocking scenario based on topographical fingerprints. In particular, for biases in the vicinity of the interference blocking regime, a flattening of the molecular image in constant height and an enhanced sensitivity of the apparent height to the operating current in the constantcurrent mode are expected. The robustness of the effect is ensured by the observation that quasidegeneracy and not exact degeneracy of the interfering many-body states is the necessary condition for the persistence of the phenomenon.

\section{ACKNOWLEDGMENTS}

We thank Prof. Jascha Repp for fruitful discussions. Moreover, we acknowledge financial support from the DFG within the research programs SPP 1243, GRK 1570, and SFB 689.

\section{APPENDIX: TUNNELING RATES AND OVERLAP INTEGRALS}

The derivation of the tunneling rates, up to a number of small differences, follows the example given in Ref. 32. In their most general form, they are given by

$$
\begin{aligned}
\Gamma_{\ell m, \ell^{\prime} m^{\prime}}^{\chi} & =\frac{2 \pi}{\hbar} \sum_{k}\left(t_{k \ell m}^{\chi}\right)^{*} t_{k \ell^{\prime} m^{\prime}}^{\chi} \delta\left(\varepsilon_{k}^{\chi}-\Delta E\right) \\
& =\varepsilon_{\ell m} \varepsilon_{\ell^{\prime} m^{\prime}} \sum_{\alpha \beta}\langle\ell m \sigma \mid \alpha \sigma\rangle M_{\alpha \beta}^{\chi}\left\langle\beta \sigma \mid \ell^{\prime} m^{\prime} \sigma\right\rangle,
\end{aligned}
$$

where

$$
M_{\alpha \beta}^{\chi}(\Delta E)=\frac{2 \pi}{\hbar} \sum_{k} \delta\left(\varepsilon_{k}^{\chi}-\Delta E\right)\langle\alpha \sigma \mid \chi \vec{k} \sigma\rangle\langle\chi \vec{k} \sigma \mid \beta \sigma\rangle .
$$

The coefficients $\langle\ell m \sigma \mid \alpha \sigma\rangle$ and the energies $\varepsilon_{\ell m}$ are obtained by diagonalizing the single-particle Hamiltonian of the molecule, which is set up by using the Slater-Koster tightbinding approximation. ${ }^{39}$ The state $|\alpha \sigma\rangle$ denotes an atomic orbital located at site $\alpha$ with position vector $\vec{R}_{\alpha}=\left(x_{\alpha}, y_{\alpha}, d\right)^{\top}$. The corresponding wave functions are approximated by the contracted Gaussian orbitals $g_{2 p}(\vec{r})$ and $g_{3 d}(\vec{r})$ to simplify the calculation of the overlap integrals. The definition of the Gaussian orbitals, their contraction coefficients $d_{i}, e_{i}$, and their exponents $a_{i}, b_{i}$ can be found in Refs. 41 and 42 . The orbitals used in this paper then are given by

$$
p_{z}(\vec{r})=n_{2 p} \vec{r} \cdot \hat{e}_{z} g_{2 p}(\vec{r}),
$$

for a $p_{z}$ orbital. A $d_{x z}$ orbital then accordingly reads

$$
d_{x z}(\vec{r})=n_{3 d} \vec{r} \cdot \hat{e}_{x} \vec{r} \cdot \hat{e}_{z} g_{3 d}(\vec{r}) .
$$

The parameters $n_{2 p}$ and $n_{3 d}$ ensure normalization. The electronic states of the tip and the substrate are given by $\mid(\chi=$ $T) \vec{k} \sigma\rangle$ and $|(\chi=S) \vec{k} \sigma\rangle$, respectively. Their wave functions 
can be expressed in the form

$$
\Psi^{\chi}(x, y, z)=\psi_{\|}^{\chi}(x, y) \psi_{\perp}^{\chi}(z)
$$

where $\psi_{\|}^{\chi}(x, y)$ is given by plane waves for $\chi=\mathrm{S}$ or by the wave function of the ground state of a two-dimensional harmonic oscillator for $\chi=\mathrm{T}$. The wave functions $\psi_{\perp}^{\chi}(z)$ are the exponentially decaying parts of the solutions of one-dimensional finite potential wells,

$$
\psi_{\perp}^{\mathrm{S}}(z)=n_{\perp}^{\mathrm{S}} e^{-\kappa_{\mathrm{S}} z} \quad \text { and } \quad \psi_{\perp}^{\mathrm{T}}(z)=n_{\perp}^{\mathrm{T}} e^{\kappa_{\mathrm{T}}\left(z-z_{\text {tip }}\right)},
$$

where $n_{\perp}^{\chi}$ accounts for normalization and $\kappa_{\chi}$ is given by

$$
\kappa_{\chi}=\sqrt{\frac{2 m}{\hbar^{2}}\left(-\varepsilon_{0}^{\chi}-\varepsilon_{z}\right)} .
$$

For the sake of reproduction, the different contributions to $M_{\alpha \beta}^{\chi}$ are listed in the following.

\section{Substrate-molecule tunneling rates}

For two $p_{z}$ orbitals located at sites $\alpha$ and $\beta, M_{\alpha \beta}^{\mathrm{S}}$ reads

$$
\begin{aligned}
M_{\alpha \beta}^{\mathrm{S}}= & \frac{4 \pi^{4}}{\hbar^{3}} n_{2 p}^{2} \sqrt{\frac{m^{3}}{2}} \sum_{i j} \frac{d_{i} d_{j}}{a_{i} a_{j}} \\
& \times \int_{0}^{\varepsilon_{F}^{\mathrm{S}}+\phi_{0}^{\mathrm{S}}} \frac{\mathrm{d} \varepsilon_{z}}{\sqrt{\varepsilon_{z}}} J_{0}\left(\tilde{k}_{\mathrm{S}}\left|\vec{R}_{\alpha \beta}\right|\right) \mathrm{e}^{-\frac{\tilde{k}_{\mathrm{S}}^{2}}{4}\left(a_{i}^{-1}+a_{j}^{-1}\right)} \\
& \times F\left(a_{i}, \kappa_{\mathrm{S}},-d\right) F\left(a_{j}, \kappa_{\mathrm{S}},-d\right)
\end{aligned}
$$

where $J_{n}(x)$ is the $n$ th-order Bessel function, $\vec{R}_{\alpha \beta}=\vec{R}_{\alpha}-\vec{R}_{\beta}$, and $\tilde{k}_{\mathrm{S}}=\sqrt{\frac{2 m}{\hbar^{2}}\left(\Delta E-\varepsilon_{0}^{\mathrm{S}}-\varepsilon_{z}\right)}$. The function $F(a, \kappa, x)$ results from the overlap of $\psi_{\perp}^{\chi}(z)$ with an atomic Gaussian orbital and is given by

$$
F(a, \kappa, x)=\frac{n_{\perp}^{\chi} e^{-a x^{2}}}{2 a}-\frac{n_{\perp}^{\chi} \kappa}{4} \sqrt{\frac{\pi}{a^{3}}} \operatorname{erfc}\left(\frac{\kappa+2 a x}{2 \sqrt{a}}\right) e^{\kappa x+\frac{\kappa^{2}}{4 a}} .
$$

Here, $\operatorname{erfc}(x)$ is the complementary error function. Consequently, it follows, for a $d_{x z}$ orbital located at $\vec{R}_{\alpha}$ and a $p_{z}$ orbital at $\vec{R}_{\beta}$, that

$$
\begin{aligned}
M_{\alpha, x z ; \beta}^{\mathrm{S}}= & -\frac{2 \pi^{4}}{\hbar^{3}} \sqrt{\frac{m^{3}}{2}} n_{3 d} n_{2 p} \cos \theta_{\alpha \beta} \sum_{i j} \frac{e_{i} d_{j}}{b_{i}^{2} a_{j}} \\
& \times \int_{0}^{\varepsilon_{F}^{\mathrm{S}}+\phi_{0}^{\mathrm{S}}} \mathrm{d} \varepsilon_{z} \frac{\tilde{k}_{\mathrm{S}}}{\sqrt{\varepsilon_{z}}} J_{1}\left(\tilde{k}_{\mathrm{S}}\left|\vec{R}_{\alpha \beta}\right|\right) \mathrm{e}^{-\frac{\tilde{k}_{\mathrm{S}}^{2}}{4}\left(b_{i}^{-1}+a_{j}^{-1}\right)} \\
& \times F\left(b_{i}, \kappa_{\mathrm{S}},-d\right) F\left(a_{j}, \kappa_{\mathrm{S}},-d\right),
\end{aligned}
$$

where $\theta_{\alpha \beta}$ is the polar angle of the planar component of the vector $\vec{R}_{\alpha \beta}$. From $M_{\alpha, x z ; \beta}^{\mathrm{S}}$ one can obtain $M_{\alpha, y z ; \beta}^{\mathrm{S}}$ by exchanging the corresponding parameters and by replacing $\cos \theta_{\alpha \beta}$ with $\sin \theta_{\alpha \beta}$. The expression $M_{\alpha, x z ; \beta, y z}^{\mathrm{S}}$ vanishes exactly due to symmetry reasons, and finally, $M_{\alpha, x z ; \beta, x z}^{\mathrm{S}}$ is given by

$$
\begin{aligned}
M_{\alpha, x z ; \beta, x z}^{\mathrm{S}}= & -\frac{\pi^{4}}{2 \hbar^{3}} \sqrt{\frac{m^{3}}{2}} n_{3 d}^{2} \int_{0}^{\varepsilon_{F}^{\mathrm{S}}+\phi_{0}^{\mathrm{S}}} \mathrm{d} \varepsilon_{z} \frac{\tilde{k}_{\mathrm{S}}^{2}}{\sqrt{\varepsilon_{z}}} \\
& \times\left[\sum_{j} \frac{e_{j}}{b_{j}^{2}} F\left(b_{j}, \kappa_{\mathrm{S}},-d\right) \mathrm{e}^{-\frac{\tilde{k}_{\mathrm{S}}^{2}}{4 b_{j}}}\right]^{2} \\
\equiv & M_{\alpha, y z ; \beta, y z}^{\mathrm{S}} .
\end{aligned}
$$

\section{Tip-molecule tunneling rates}

Due to the fact that the planar energy component of the tip wave function is fixed at $\varepsilon_{\|}=\hbar \omega$, there is only one single integration in energy to evaluate in order to obtain the tip-molecule tunneling rates. Because of this, they are much more straightforward to calculate than their substrate-molecule counterparts:

$$
\begin{aligned}
M_{\alpha \beta}^{T} & =\frac{2 \pi}{\hbar^{2}} \int_{0}^{-\varepsilon_{0}^{\mathrm{T}}} \mathrm{d} \varepsilon_{z} D\left(\varepsilon_{z}\right)\langle\alpha \sigma \mid T \vec{k} \sigma\rangle\langle T \vec{k} \sigma \mid \beta \sigma\rangle \delta\left(\varepsilon_{k}^{\mathrm{T}}-\Delta E\right) \\
& =\frac{2 \pi}{\hbar^{2}} \sqrt{\frac{m}{2}} \frac{L_{\text {tip }}}{\Delta E-\varepsilon_{0}^{T}-\hbar \omega}\langle\alpha \sigma \mid T \vec{k} \sigma\rangle\langle T \vec{k} \sigma \mid \beta \sigma\rangle . \text { (A12) }
\end{aligned}
$$

The parameter $L_{\text {tip }}$ stems from the one-dimensional density of states of the tip and it is canceled later on by the normalization of the tip wave function. Another effect of the single integration in energy is that all possible combinations of $p_{z}, d_{x z}$, and $d_{y z}$ orbitals are surviving. In order not to go beyond the constraints of this paper, we only list the overlap integrals needed to construct the matrices $M_{\alpha \beta}^{\mathrm{T}}$. After introducing the following parameters and abbreviations $-v^{2}=\frac{m \omega}{2 \hbar}, \Delta y_{\alpha}=y_{\text {tip }}-y_{\alpha}$, $\Delta x_{\alpha}=x_{\text {tip }}-x_{\alpha}$, and, finally, $\kappa_{\mathrm{T}}=\sqrt{\frac{2 m}{\hbar^{2}}(\hbar \omega-\Delta E)}$-we are able to give the overlap integrals between the different orbitals located at $\vec{R}_{\alpha}$ and the tip wave function:

$$
\begin{aligned}
\langle\alpha \sigma \mid \mathrm{T} \vec{k} \sigma\rangle= & -n_{2 p} \sqrt{2 \pi} v \sum_{j} \frac{d_{j}}{a_{j}+v^{2}} \\
& \times \exp \left(-\frac{v^{2} a_{j}}{v^{2}+a_{j}}\left(\Delta x_{\alpha}^{2}+\Delta y_{\alpha}^{2}\right)\right) \\
& \times F\left(a_{j}, \kappa_{\mathrm{T}}, d-z_{\text {tip }}\right)
\end{aligned}
$$

$$
\begin{aligned}
\left\langle\alpha_{x z} \sigma \mid \mathrm{T} \vec{k} \sigma\right\rangle= & n_{3 d} \sqrt{2 \pi} v^{3} \sum_{j} \frac{e_{j} \Delta x_{\alpha}}{\left(b_{j}+v^{2}\right)^{2}} \\
& \times \exp \left(-\frac{v^{2} a_{j}}{v^{2}+a_{j}}\left(\Delta x_{\alpha}^{2}+\Delta y_{\alpha}^{2}\right)\right) \\
& \times F\left(a_{j}, \kappa_{\mathrm{T}}, d-z_{\text {tip }}\right), \\
\left\langle\alpha_{y z} \sigma \mid \mathrm{T} \vec{k} \sigma\right\rangle= & n_{3 d} \sqrt{2 \pi} v^{3} \sum_{j} \frac{e_{j} \Delta y_{\alpha}}{\left(b_{j}+v^{2}\right)^{2}} \\
& \times \exp \left(-\frac{v^{2} a_{j}}{v^{2}+a_{j}}\left(\Delta x_{\alpha}^{2}+\Delta y_{\alpha}^{2}\right)\right) \\
& \times F\left(a_{j}, \kappa_{\mathrm{T}}, d-z_{\text {tip }}\right) .
\end{aligned}
$$


*Corresponding author: andrea.donarini@ physik.uni-r.de

${ }^{1}$ L. Esaki, Phys. Rev. 109, 603 (1958).

${ }^{2}$ I. W. Lyo and P. Avouris, Science 245, 1369 (1989).

${ }^{3}$ P. Bedrossian, D. M. Chen, K. Mortensen, and J. A. Golov-chenko, Nature 342, 258 (1989).

${ }^{4}$ J. Chen, M. A. Reed, A. M. Rawlett, and J. M. Tour, Science 286, 1550 (1999).

${ }^{5}$ C. Zeng, H. Wang, B. Wang, J. Yang, and J. G. Hou, Appl. Phys. Lett. 77, 3595 (2000).

${ }^{6}$ M. Rinkiö, A. Johansson, V. Kotimäki, and P. Törmä, ACS Nano 4, 3356 (2010).

${ }^{7}$ K. J. Franke, G. Schulze, N. Henningsen, I. Fernández-Torrente, J. I. Pascual, S. Zarwell, K. Rück-Braun, M. Cobian, and N. Lorente, Phys. Rev. Lett. 100, 036807 (2008).

${ }^{8}$ Y. Xue, S. Datta, S. Hong, R. Reifenberger, J. I. Henderson, and C. P. Kubiak, Phys. Rev. B 59, R7852 (1999).

${ }^{9}$ M. Grobis, A. Wachowiak, R. Yamachika, and M. F. Crommie, Appl. Phys. Lett. 86, 204102 (2005).

${ }^{10}$ X. W. Tu, G. R. Mikaelian, and W. Ho, Phys. Rev. Lett. 100, 126807 (2008).

${ }^{11}$ L. Chen, Z. Hu, A. Zhao, B. Wang, Y. Luo, J. Yang, and J. G. Hou, Phys. Rev. Lett. 99, 146803 (2007).

${ }^{12}$ X. Q. Shi, W. W. Pai, X. D. Xiao, J. I. Cerdá, R. Q. Zhang, C. Minot, and M. A. Van Hove, Phys. Rev. B 80, 075403 (2009).

${ }^{13}$ B. W. Heinrich, M. V. Rastei, D.-J. Choi, T. Frederiksen, and L. Limot, Phys. Rev. Lett. 107, 246801 (2011).

${ }^{14}$ J. Gaudioso, L. J. Lauhon, and W. Ho, Phys. Rev. Lett. 85, 1918 (2000).

${ }^{15}$ N. Traverso Ziani, G. Piovano, F. Cavaliere, and M. Sassetti, Phys. Rev. B 84, 155423 (2011).

${ }^{16}$ D. V. Cardamone, C. A. Stafford, and S. Mazumdar, Nano Lett. 6, 2422 (2006).

${ }^{17}$ S.-H. Ke, W. Yang, and U. Baranger, Nano Lett. 8, 3257 (2008).

${ }^{18}$ Z. Qian, R. Li, X. Zhao, S. Hou, and S. Sanvito, Phys. Rev. B 78, 113301 (2008).

${ }^{19}$ G. Begemann, D. Darau, A. Donarini, and M. Grifoni, Phys. Rev. B 77, 201406(R) (2008); 78, 089901(E) (2008).

${ }^{20}$ G. C. Solomon, D. Q. Andrews, T. Hansen, R. H. Goldsmith, M. R. Wasielewski, R. P. V. Duyne, and M. A. Ratner, J. Chem. Phys. 129, 054701 (2008).

${ }^{21}$ D. Darau, G. Begemann, A. Donarini, and M. Grifoni, Phys. Rev. B 79, 235404 (2009).
${ }^{22}$ A. Donarini, G. Begemann, and M. Grifoni, Nano Lett. 9, 2897 (2009).

${ }^{23}$ A. Donarini, G. Begemann, and M. Grifoni, Phys. Rev. B 82, 125451 (2010).

${ }^{24}$ T. Markussen, R. Stadler, and K. S. Thygesen, Nano Lett. 10, 4260 (2010).

${ }^{25}$ Y. Tsuji, A. Staykov, and K. Yoshizawa, J. Am. Chem. Soc. 133, 5955 (2011).

${ }^{26}$ T. Markussen, R. Stadler, and K. S. Thygesen, Phys. Chem. Chem. Phys. 13, 14311 (2011).

${ }^{27}$ M. J. Ernzerhof, Chem. Phys. 135, 014104 (2011).

${ }^{28}$ M. Mayor, H. B. Weber, J. Reichert, M. Elbing, C. von Hanisch, D. Beckmann, and M. Fischer, Angew. Chem. Int. Ed. 42, 5834 (2003).

${ }^{29}$ M. Taniguchi, M. Tsutsui, R. Mogi, T. Sugawara, Y. Tsuji, K. Yoshizawa, and T. Kawai, J. Am. Chem. Soc. 133, 11426 (2011).

${ }^{30}$ S. V. Aradhya, J. S. Meisner, M. Krikorian, S. Ahn, R. Parameswaran, M. L. Steigerwald, C. Nuckolls, and L. Venkataraman, Nano Lett. 12, 1643 (2012).

${ }^{31}$ C. M. Guédon, H. Valkenier, T. Markussen, K. S. Thygesen, J. C. Hummelen, and S. J. van der Molen, Nature Nanotech. 7, 305 (2012).

${ }^{32}$ S. Sobczyk, A. Donarini, and M. Grifoni, Phys. Rev. B 85, 205408 (2012).

${ }^{33}$ J. Repp, G. Meyer, S. M. Stojkovic, A. Gourdon, and C. Joachim, Phys. Rev. Lett. 94, 026803 (2005).

${ }^{34}$ P. Liljeroth, J. Repp, and G. Meyer, Science 317, 1203 (2007).

${ }^{35}$ I. Fernández-Torrente, D. Kreikemeyer-Lorenzo, A. Stróźecka, K. J. Franke, and J. I. Pascual, Phys. Rev. Lett. 108, 036801 (2012).

${ }^{36} \mathrm{~K}$. Blum, Density matrix Theory and Applications (Plenum Press, New York, 1996).

${ }^{37}$ U. Weiss, Quantum Dissipative Systems (World Scientific, Singapore, 2012), 4th ed.

${ }^{38}$ M. Grifoni, M. Sassetti, and U. Weiss, Phys. Rev. E 53, R2033 (1996).

${ }^{39}$ J. C. Slater and G. F. Koster, Phys. Rev. B 94, 1498 (1954).

${ }^{40}$ T. Sonnleitner, I. Swart, N. Pavliček, A. Pöllmann, and J. Repp, Phys. Rev. Lett. 107, 186103 (2011).

${ }^{41}$ W. J. Hehre, R. F. Stewart, and J. A. Pople, J. Chem. Phys. 51, 2657 (1969).

${ }^{42}$ W. J. Pietro, B. A. Levi, W. J. Hehre, and R. F. Stewart, Inorg. Chem. 19, 2225 (1980). 